\title{
Size of emptied plaque cavity following spontaneous rupture is related to coronary dimensions, not to the degree of lumen narrowing. A study with intravascular ultrasound in vivo
}

C von Birgelen, W Klinkhart, G S Mintz, H Wieneke, D Baumgart, M Haude, T Bartel, S Sack, J Ge, R Erbel

\begin{abstract}
Objective-To identify any potential relations between the size of an emptied plaque cavity and the remodelling pattern, plaque or vessel dimensions, lumen narrowing, and other ultrasonic lesion characteristics.

Design-Intravascular ultrasound was used to examine prospectively 51 ruptured ulcerated coronary plaques. Cross sectional area measurements comprised lumen, vessel, plaque, and emptied plaque cavity. Lumen narrowing was calculated as $1-$ (lesion lumen area/reference lumen area) $\times 100 \%$. A remodelling index was calculated as lesion vessel area/reference vessel area, and plaques were divided into those with values $>1.05$ (group A) and $\leqslant 1.05$ (group B).

Results-Of the total of 51 plaques, 36 (71\%) were assigned to group A and $15(29 \%)$ to group $B$. In neither group was there a significant difference in reference dimensions and lumen narrowing. However, lesion vessel (mean (SD): $22.6(8.1) \mathrm{mm}^{2} v 17.5(4.3) \mathrm{mm}^{2} ; \mathrm{p}=0.006$ ) and plaque areas $\left(15.8(6.2) \mathrm{mm}^{2} v 12.8(3.2) \mathrm{mm}^{2} ; \mathrm{p}=0.03\right)$ were greater in group A than in group B. The cavity inside the plaque was larger in group A than in group B $\left(2.8(1.6) \mathrm{mm}^{2} v 1.8(0.9) \mathrm{mm}^{2}\right.$; $\mathrm{p}=0.007)$ and showed a positive linear relation with lesion and reference vessel size $(r=0.58$ and 0.56 , respectively; $\mathrm{p}<0.001$ ), but not with lumen narrowing.

Conclusions-The size of the emptied cavity inside ruptured plaques is on average larger in lesions with adaptive vascular remodelling, and shows a linear relation with lesion plaque and vessel size and with the reference dimensions, but not with the degree of lumen narrowing.

(Heart 2000;84:483-488)
\end{abstract}

Keywords: intravascular ultrasound; ultrasonic scanning; plaque rupture; remodelling

Much information about fissuring and disruption of atherosclerotic coronary plaques, the trigger of acute coronary syndromes, has been obtained from necrospy data, ${ }^{1-8}$ and it has been shown that plaque rupture results in thrombus formation and often in emptying of the atheromatous core. Angiographic techniques provide data on the size and shape of the luminal silhouette in vivo. ${ }^{910}$ However, the value of angiography for the assessment of atherosclerosis is limited, as compensatory vascular enlargement may accompany plaque accumulation and partly accommodate the increasing plaque burden, ${ }^{11-13}$ thereby preserving lumen dimensions and causing angiographic underestimation of the underlying atherosclerosis.

Intravascular ultrasound allows transmural visualisation of coronary vessels and has been most useful in confirming the concept of compensatory vascular enlargement in vivo. ${ }^{14-19}$ Along with its use in assessing vascular remodelling, ${ }^{20-27}$ intravascular ultrasound can also be employed to confirm the presence of plaque rupture in vivo and to measure the size of the emptied plaque cavity (fig 1). ${ }^{28-34}$ In patients with unstable angina and also in those with plaque rupture documented by intravascular ultrasound, a greater degree of vascular remodelling has been described; this is associ- ated with larger plaque and vessel cross sectional areas. ${ }^{27}{ }^{33}$ In this study we analysed ruptured plaques using intravascular ultrasound in order to evaluate whether the remodelling pattern, plaque or vessel size, or other ultrasonic lesion characteristics might be related to the size of the cavity inside the ruptured plaque.

\section{Methods}

PATIENT POPULATION

The study population consisted of 51 patients with ulcerated ruptured plaques confirmed on intravascular ultrasound (lesion type VI-a according to a recently suggested intravascular ultrasound lesion classification ${ }^{19}$ ). The lesions were all in previously untreated coronary arteries. This consecutive series of ruptured plaques was identified prospectively from among our patients with unstable angina or with an angiographic picture suspicious of plaque rupture. All plaques included in this study met the following intravascular ultrasound criteria:

- high quality images of the lesion as well as of the proximal and distal reference segments;

- non-ostial lesion location;

- calcification did not limit quantitative assessment of the vessel area; 

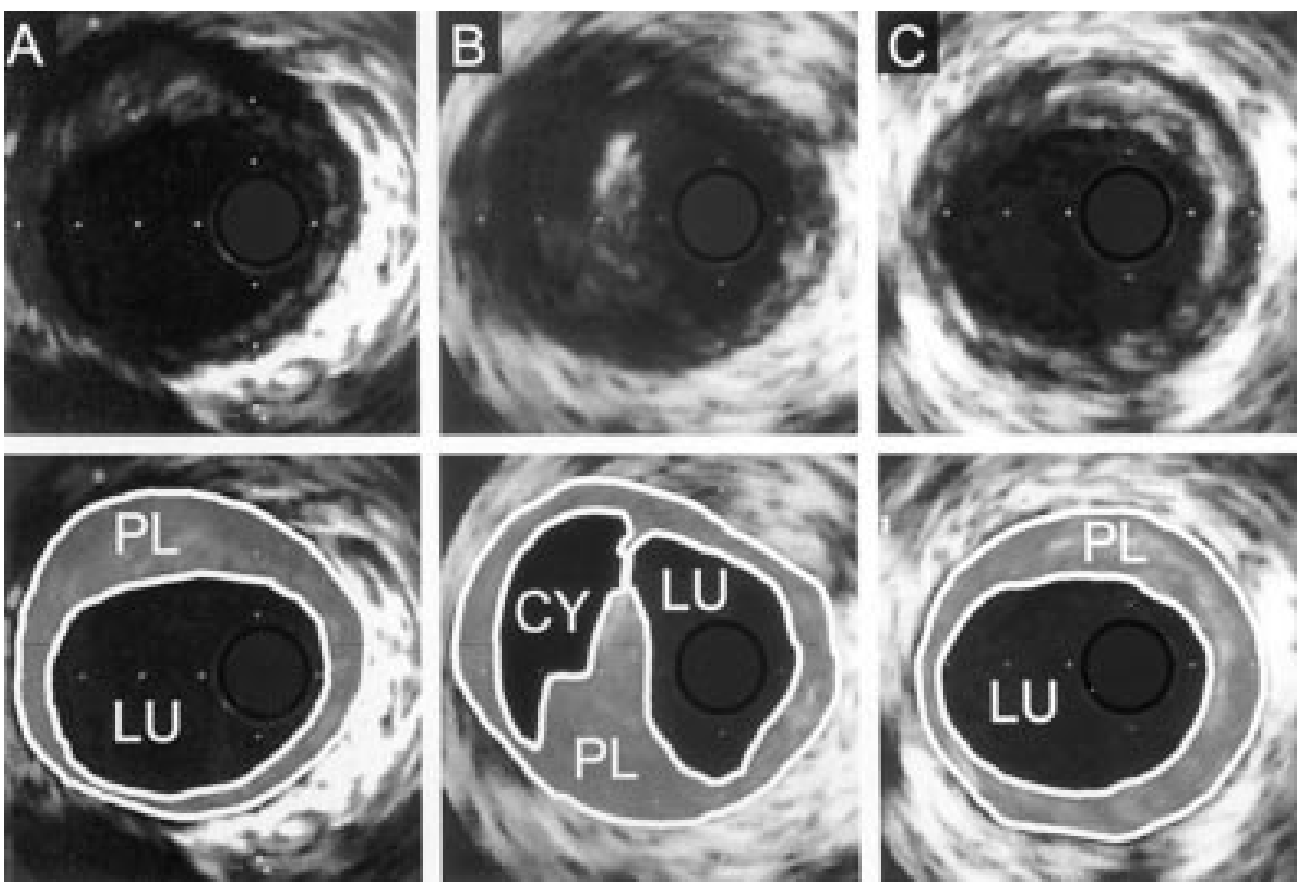

Figure 1 Intravascular ultrasound planimetry in ruptured right coronary plaque of a patient in group $A$ (remodelling index > 1.05). Lumen (LU), plaque (PL), plaque cavity $(C Y)$, and vessel area (within external contour) were measured in the proximal $(A)$ and distal reference segment $(C)$, and at the lesion site $(B)$. Lesion and mean reference vessel cross sectional area measured $24.4 \mathrm{~mm}^{2}$ and $22.25 \mathrm{~mm}^{2}$, respectively; the remodelling index was 1.09 . Plaque cavity cross sectional area measured $4.6 \mathrm{~mm}^{2}$. Lumen area narrowing was $37 \%$.

- absence of side branches between the lesion and the proximal and distal reference segments;

- absence of very angulated segments on angiography.

The study was approved by the local Council on Human Research, and all patients signed a written informed consent form, approved by the local medical ethics committee.

INTRAVASCULAR ULTRASOUND IMAGING AND INTERVENTIONAL PROCEDURE

All patients were being treated with aspirin and received heparin intravenously before the study. Procedures were performed by the femoral approach, using adequately sized standard $8 \mathrm{~F}$ guiding catheters and 0.014 inch $(0.36 \mathrm{~mm})$ standard or extra support coronary guide wires.

Intravascular ultrasound imaging was performed before any intervention and after an intracoronary bolus injection of $200 \mu \mathrm{g}$ of glyceryl trinitrate.

The intravascular ultrasound studies were performed with two commercially available systems. The first system was a mechanical sector scanner (UltraView ${ }^{\mathrm{TM}}$, Boston Scientific Corporation, San Jose, California, USA) which incorporated $30 \mathrm{MHz}$ single element bevelled transducers. The second system was a solid state device (InVision ${ }^{\mathrm{TM}}$, Endosonics, Rancho Cordova, California, USA) which used $3.0 \mathrm{~F}$ electronic monorail catheters. With both systems, the transducer was withdrawn by use of motorised pullback devices to perform the imaging sequence. ${ }^{34}$ All transducer pullbacks were started as distal as possible, and the entire artery was imaged to the aorto-ostial junction. In addition, the site of the plaque rupture was examined using manual interrogation. We performed bolus injections of dye and saline to confirm (or exclude) the presence of plaque rupture, as previously described. ${ }^{34}$ All intravascular ultrasound examinations were recorded on 0.5 inch $(1.27 \mathrm{~cm})$ high resolution super VHS tape for off-line analysis. There was no procedural or postprocedural in-hospital complication that could possibly have been related to intravascular ultrasound or to the interventional procedure.

INTRAVASCULAR ULTRASOUND IMAGE ANALYSIS All intravascular ultrasound runs were analysed by an experienced analyst and overread by two experienced cardiologists. Validation of plaque

Table 1 Clinical characteristics of the whole study population and of groups $A$ and $B$

\begin{tabular}{|c|c|c|c|}
\hline Variable & $\begin{array}{l}\text { All patients } \\
(n=51)\end{array}$ & $\begin{array}{l}\text { Group } A \\
(n=36)\end{array}$ & $\begin{array}{l}\text { Group } B \\
(n=15)\end{array}$ \\
\hline Male sex & $48(94)$ & $34(94)$ & $14(93)$ \\
\hline Age (years) (mean (SD)) & $59(11)$ & $60(10)$ & $57(13)$ \\
\hline \multicolumn{4}{|l|}{ Risk factors } \\
\hline Hypercholesterolaemia & $46(90)$ & $34(94)$ & $12(80)$ \\
\hline Hypertension & $33(65)$ & $24(67)$ & $9(60)$ \\
\hline Smoking & $29(57)$ & $23(64)$ & $6(40)$ \\
\hline Diabetes mellitus & $6(12)$ & $6(17)$ & $0(0)$ \\
\hline Family history & $29(57)$ & $23(64)$ & $6(40)$ \\
\hline \multicolumn{4}{|l|}{ History } \\
\hline $\begin{array}{l}\text { Previous PTCA of } \\
\text { another vessel }\end{array}$ & $13(25)$ & $9(25)$ & $4(27)$ \\
\hline $\begin{array}{l}\text { Previous myocardial } \\
\text { infarction }\end{array}$ & $27(53)$ & $22(61)$ & $5(33)$ \\
\hline \multicolumn{4}{|l|}{ Angina } \\
\hline Stable angina & $9(18)$ & $5(14)$ & $4(27)$ \\
\hline Unstable angina & $42(82)$ & $31(86)$ & $11(73)$ \\
\hline -Braunwald class I B & $4(8)$ & $2(6)$ & $2(13)$ \\
\hline -Braunwald class II B & $10(20)$ & $8(22)$ & $3(20)$ \\
\hline -Braunwald class III B & $28(57)$ & $21(41)$ & $6(40)$ \\
\hline
\end{tabular}

Data are n (\%) except where stated. Differences were not statistically significant. 
Table 2 Quantitative intravascular ultrasound data

\begin{tabular}{|c|c|c|c|c|}
\hline Variable & $\begin{array}{l}\text { All patients } \\
(n=51)\end{array}$ & $\begin{array}{l}\text { Group } A \\
(n=36)\end{array}$ & $\begin{array}{l}\text { Group } B \\
(n=15)\end{array}$ & $\begin{array}{l}p \text { Value } \\
(A v B)\end{array}$ \\
\hline \multicolumn{5}{|l|}{ Lesion } \\
\hline Lumen area $\left(\mathrm{mm}^{2}\right)$ & $6.2(3.4)$ & 6.8 (3.7) & $4.7(1.8)$ & 0.008 \\
\hline Vessel area $\left(\mathrm{mm}^{2}\right)$ & $21.1(7.6)$ & $22.6 \quad(8.1)$ & $17.5 \quad(4.3)$ & 0.006 \\
\hline Plaque plus media area $\left(\mathrm{mm}^{2}\right)$ & $14.9(5.6)$ & $15.8 \quad(6.2)$ & $12.8 \quad(3.2)$ & 0.03 \\
\hline Plaque cavity area $\left(\mathrm{mm}^{2}\right)$ & $2.5 \quad(1.5)$ & $2.8(1.6)$ & $1.8(0.9)$ & 0.007 \\
\hline \multicolumn{5}{|l|}{ Reference } \\
\hline Lumen area $\left(\mathrm{mm}^{2}\right)$ & 9.9 (3.6) & $10.4(3.6)$ & $8.6(3.6)$ & NS \\
\hline Vessel area $\left(\mathrm{mm}^{2}\right)$ & $18.9(5.9)$ & $19.3(6.4)$ & $17.9(4.5)$ & NS \\
\hline Plaque plus media area $\left(\mathrm{mm}^{2}\right)$ & $9.0 \quad(3.9)$ & $8.9(4.4)$ & $9.3(2.4)$ & NS \\
\hline \multicolumn{5}{|l|}{ Lesion-reference relation } \\
\hline Lumen area narrowing (\%) & $34.1(27.7)$ & $31.1(29.9)$ & $41.2(20.3)$ & NS \\
\hline Remodelling index & $1.11(0.14)$ & $1.17(0.13)$ & $0.98(0.07)$ & $<0.001$ \\
\hline
\end{tabular}

Data are mean (SD). slice that was analysed had the smallest lumen area. ${ }^{1522}$ If there were several image slices with an equal lumen area, the one with the largest plaque cavity was analysed. In general, the largest plaque cavity area was observed at (or close to) the slice of the smallest lumen area.

Lesion plaque composition was assessed visually as previously described. ${ }^{18}$ The total arc of calcium was measured with a protractor centred on the lumen. Thrombus formation was a heterogeneously reflecting plaque with layering, ${ }^{32}$ oscillating or undulating fine speckles within the plaque, ${ }^{18}$ and a rough intimal surface or a negative imprint of the intravascular ultrasound catheter. ${ }^{29}$

Reference segments were analysed in a similar way to the lesion. The proximal and distal reference segments were the most normal looking cross sections (smallest plaque plus media) $\leqslant 10 \mathrm{~mm}$ distal and proximal to the lesion. This method of reference segment selection has been published previously. ${ }^{22}$ The use of motorised pullbacks helped to limit the axial distance between the lesion and the reference segments. The reference lumen, vessel, and plaque plus media areas reported were the average of the proximal and distal reference.

Lesion lumen area at the minimum lumen site was compared with the reference lumen area; lumen area narrowing was calculated as (1-(lesion lumen area/reference lumen area)) $\times 100 \%$. Reduction in the lesion lumen area could theoretically result from plaque plus media increase or vessel area decrease. To study vascular remodelling, we compared the
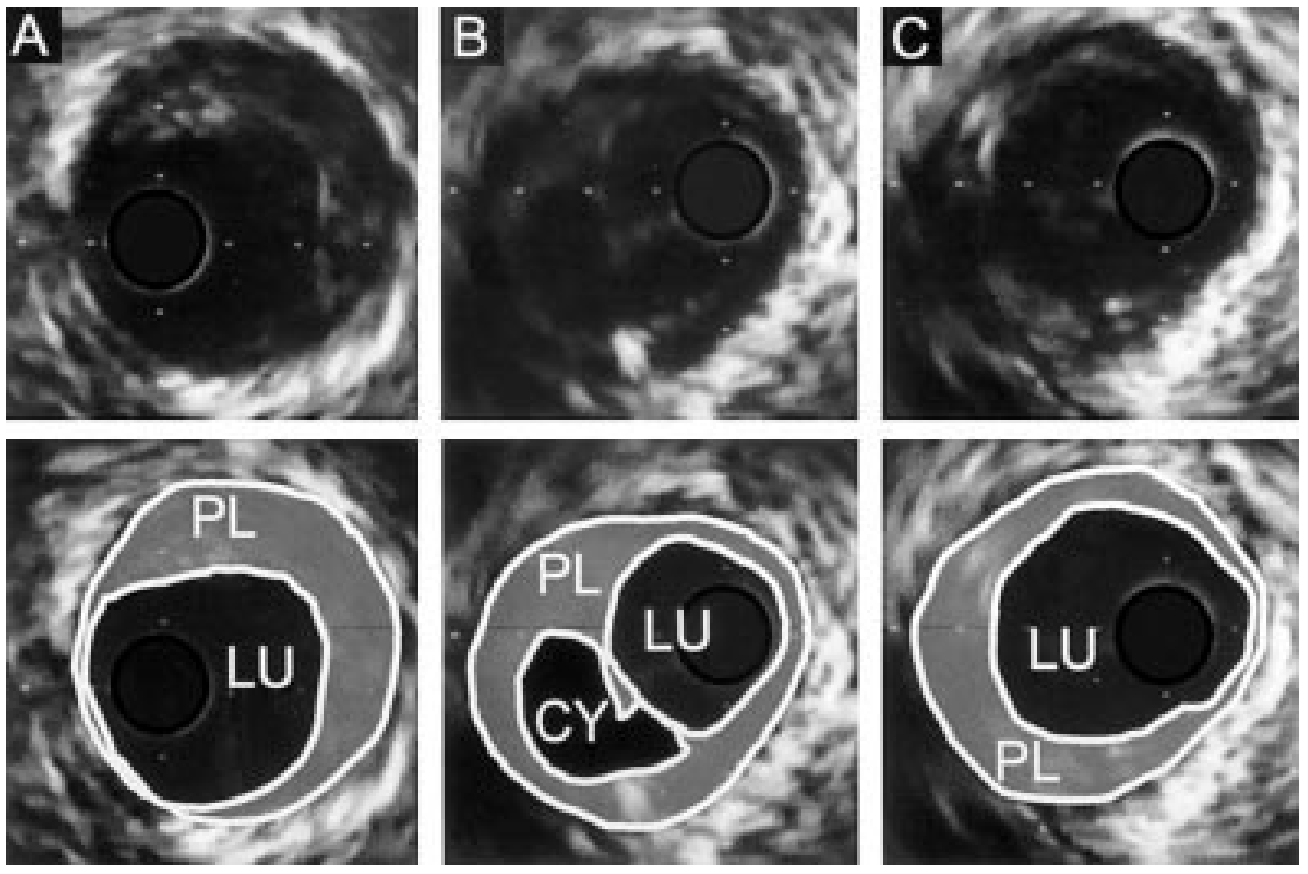

Figure 2 Example of ruptured right coronary plaque from a patient in group $B$ (remodelling index $\leqslant 1.05$ ). Lumen $(L U)$, plaque (PL), plaque cavity (CY), and vessel area (within external contour) were measured in the proximal $(A)$ and distal reference segment $(C)$, and at lesion site $(B)$. Remodelling index was 0.97. Lesion and mean reference vessel area measured $15.2 \mathrm{~mm}^{2}$ and $15.7 \mathrm{~mm}^{2}$, respectively. Plaque cavity area was $2.6 \mathrm{~mm}^{2}$ and lumen area narrowing measured 40\%. Despite comparable lumen area narrowing in the two examples in figs 1 and 2, plaque cavity area was significantly smaller in the present example, which showed smaller vascular dimensions than the vessel segment of fig 1 (note the difference in the $1 \mathrm{~mm}$ calibration grid in figs 1 and 2). 

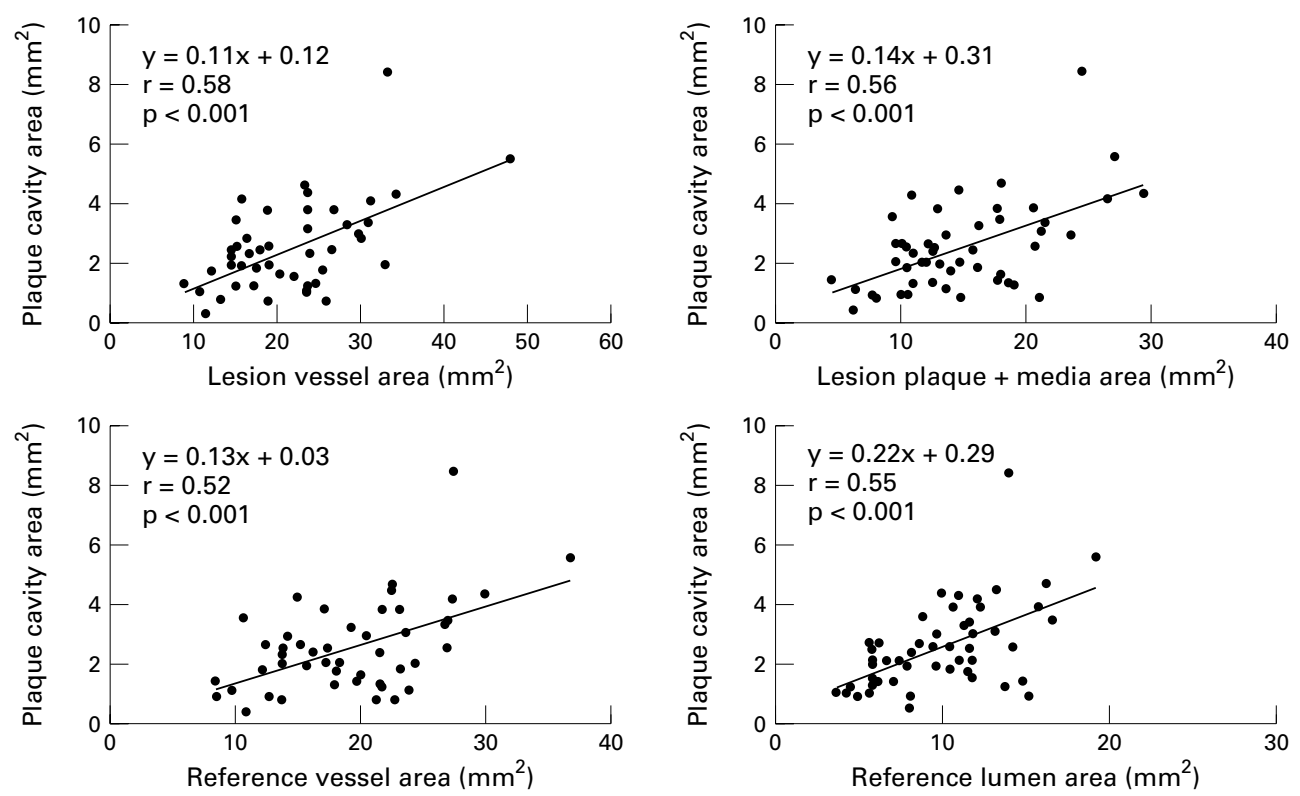

Figure 3 Linear regression analyses comparing vascular dimensions with plaque cavity size. The emptied cavity inside the ruptured plaque showed a significant linear relation with lesion plaque and vessel size (upper panels), and with the reference dimensions (lower panels).

lesion vessel area with the reference vessel area to produce a remodelling index, defined as lesion vessel area/reference vessel area. Based on the value of this index, we divided the plaques into two groups: group A, with values $>1.05$, and group $\mathrm{B}$, with values $\leqslant 1.05$.

\section{STATISTICAL ANALYSIS}

Quantitative data are given as mean values (SD). Dichotomous variables are expressed as frequencies; categorical data are compared using $\chi^{2}$ statistics. Continuous variables are compared using the two tailed Student $t$ test and linear regression analysis. Probability values of $\mathrm{p}<0.05$ are considered significant.

\section{Results}

REMODELLING STATE

A remodelling index of $>1.05$ was found to be present in $36(71 \%)$ of 51 lesions, which were assigned to group A. The other 15 lesions $(29 \%)$ had a remodelling index of $\leqslant 1.05$ (group B).

CLINICAL DATA AND LESION CHARACTERISTICS Lesions were located in the left anterior descending $(\mathrm{n}=23(45 \%))$, right $(\mathrm{n}=24$

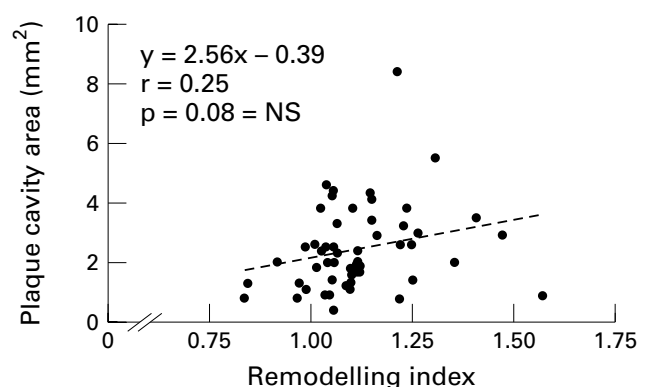

$(47 \%))$, left circumflex $(n=3(6 \%))$, and left main coronary arteries $(n=1(2 \%))$, in the proximal $(n=18 \quad(35 \%)), \quad \operatorname{mid} \quad(n=24$ $(47 \%))$, and distal segments $(n=9(18 \%))$. There was a non-significant trend for group A patients to have suffered more often from previous myocardial infarction and to be more likely to have unstable angina (table 1). In groups $\mathrm{A}$ and $\mathrm{B}$, lesions were located in the left anterior descending (15/36 (42\%) $v 8 / 15$ $(53 \%))$, right $(18 / 36(50 \%) v 6 / 15(40 \%))$, left circumflex $(2 / 36(6 \%) v 1 / 15(7 \%))$, and left main coronary arteries $(1 / 36(3 \%) v 0 / 15$ $(0 \%))$, in the proximal $(13 / 36(36 \%) v 5 / 15$ $(33 \%))$, mid $(19 / 36(53 \%)$ v $5 / 15(33 \%))$, and distal segments $(4 / 36$ (11\%) $v \quad 5 / 15$ $(33 \%))$.

Predominantly soft plaque composition was found in $33 \%$ of all lesions (17 of 51 ; groups A $v$ B 12/36 v 5/15). Some calcium was found in 26 lesions $(51 \%)$, and the total arc of calcium at lesion site (mean (SD)) was $43(55)^{\circ}$. Thrombus formation was observed in 15 lesions (29\%). There was a trend towards a larger total arc of lesion site calcium in group A than in group B $\left(50(60)^{\circ}\right.$ v $\left.26(38)^{\circ} ; \mathrm{p}=0.09\right)$.

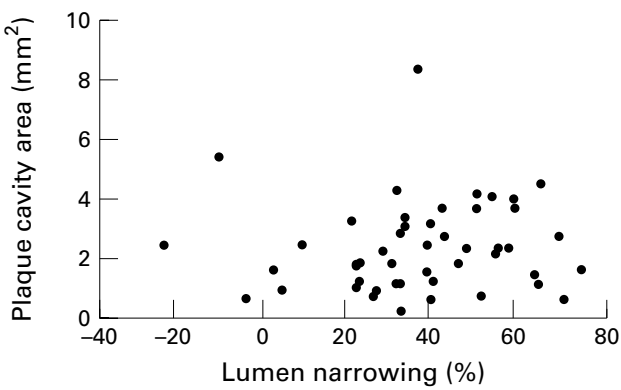

Figure 4 Comparison of remodelling index and lumen narrowing with plaque cavity size. The emptied cavity inside the ruptured plaque showed a significant linear relation with lesion plaque and vessel size, and with reference dimensions. There was no significant relation between plaque cavity size and remodelling index (left panel) or the per cent lumen area narrowing (right panel, note scattered distribution of data points). 
QUANTITATIVE INTRAVASCULAR ULTRASOUND DATA

In group $A$, the lesion vessel area was larger than the reference vessel area (22.6 (8.1) $v 19.3$ (6.4) $\left.\mathrm{mm}^{2}, \mathrm{p}<0.001\right)$, while in group $\mathrm{B}$ the vessel area at lesion site did not differ significantly from the reference (17.5 (4.3) $v$ 17.9 (4.5) $\mathrm{mm}^{2}$, NS) (table 2).

The cavity inside the plaque was larger in group A than in group B (2.8 (1.6) $v 1.8(0.9)$ $\left.\mathrm{mm}^{2} ; \mathrm{p}=0.007\right)$. Examples are given in figs 1 and 2 . There were positive linear relations between plaque cavity area and lesion vessel and plaque areas, and between plaque cavity area and reference vessel and lumen areas (fig 3) $(r=0.52-0.58 ; \mathrm{p}<0.001)$. There was no significant relation between plaque cavity area and either per cent lumen narrowing or remodelling index (fig 4 ).

\section{Discussion}

Various studies have shown that ruptured plaques can be detected satisfactorily by intravascular ultrasound. ${ }^{28} 313334$ In the present study, we also evaluated compensatory vascular enlargement (that is, adaptive vascular remodelling), which is the increase in vessel area that occurs to compensate for the increasing plaque burden during the early phase of plaque accumulation. In addition, we measured the size of the cavity inside ruptured ulcerated plaques by intravascular ultrasound and found good agreement with data reported in previous studies. $^{33} 34$

The current in vivo study shows that the size of the emptied plaque cavity following plaque rupture is linearly related to the absolute lesion plaque and vessel dimensions, and to the reference dimensions. In addition, plaque cavity size was larger in the lesions in group A (with a remodelling index $>1.05$ ), which were present in $71 \%$ of the patients. However, neither lumen narrowing nor remodelling index showed a direct relation with plaque cavity size; thus these variables do not allow one to identify plaques at increased risk of massive atheroma embolisation in the case of plaque rupture with ulceration.

The larger mean plaque cavity size in group A lesions can easily be explained by the relatively larger lesion plaque and vessel dimensions in cases of greater adaptive remodelling. However, a linear relation between plaque cavity size and remodelling index was not found and should not be expected. Adaptive remodelling with higher compensatory vascular enlargement is associated with relatively larger plaque and vessel dimensions at the lesion site, but this remodelling pattern can be found in vascular segments of any (reference) size, which renders a linear relation with plaque cavity size improbable.

INSIGHTS PROVIDED BY NECROPSY STUDIES Previous necropsy studies have underlined the importance of plaque size in the development of a large atheromatous core and plaque rupture, suggesting that plaque rupture with thrombus formation is mostly associated with significant plaque burden. ${ }^{146}$ Most pathoana- tomical studies have suggested that the atheromatous core enlarges with plaque growth. ${ }^{33-37}$ However, comparison of plaque size and core size of mostly non-ruptured plaques showed no linear relation. ${ }^{8}$ Our observation in the present study that the cavity inside the plaque showed no relation to percentage lumen narrowing corroborates previous angiographic data and pathoanatomical findings. ${ }^{89}$

Rupture and ulceration of lesions with large vessel dimensions and a marked atheromatous core may be followed by embolisation of more atheromatous material, leading to microinfarction, exposure of more thrombogenic tissue, ${ }^{6}$ and (most probably) a worse clinical outcome. This may partly explain the high representation of plaques with large plaque burden that was described in previous studies on patients with fatal plaque rupture. ${ }^{14^{6}}$ Conversely, intravascular ultrasound studies in vivo imply the assessment of survivors of the event. Therefore, histological and intravascular ultrasound studies may be complementary in improving our understanding of coronary atherosclerosis, acute coronary syndromes, and plaque rupture.

PREVIOUS INTRAVASCULAR ULTRASOUND STUDIES Adaptive remodelling with compensatory vascular enlargement was previously identified as the predominant remodelling pattern of unstable or ruptured plaques. ${ }^{26} 2733$ Gyöngyösi et al reported adaptive remodelling in unstable patients in no more than $37 \%$ of all plaques, ${ }^{27}$ while adaptive remodelling was found in 16 of 26 ruptured plaques (62\%). Moriuchi et al observed compensatory vascular enlargement in 11 of 22 ruptured plaques $(50 \%))^{33}$

LIMITATIONS AND POTENTIAL SOURCES OF ERROR As with all histological and most intravascular ultrasound studies, our study provides only a snapshot view of coronary artery disease, and because ostial and extremely calcified plaques were not included, our findings may not be applicable to these lesions. Visual assessment of plaque composition is limited, and ultrasonic tissue classification does not necessarily correspond to histology. Remodelling of the reference segments may affect assessment of lesion site remodelling. We may also have missed some ruptured plaques in which the cavity was extremely small, filled by thrombus, or sealed by reattachment of the flap. In addition, angiographic suspicion of plaque rupture may have triggered intravascular ultrasound examination in several cases. Accordingly, we cannot exclude a certain selection bias towards larger plaque cavities in this population. Finally, plaque rupture may lead to haemorrhage and thrombus formation, which could alter plaque geometry and the remodelling pattern. However, observation of a predominance of greater compensatory vascular enlargement in ruptured plaques in vivo is supported by the in vitro data of Pasterkamp et al, ${ }^{38}$ who found an association between histopathological markers of plaque vulnerability and adaptive remodelling in plaques that had not undergone plaque rupture. 
CONCLUSIONS

The size of the emptied cavity inside the ruptured plaque is on average larger in lesions with adaptive vascular remodelling, and shows a linear relation with lesion plaque and vessel size and with reference dimensions, but not with the degree of lumen narrowing.

This project was supported by a grant of the German Research Society, DFG, Bonn, Germany (DFG $1554-1$ ).

1 Falk E. Plaque rupture with severe pre-existing stenosis precipitating coronary thrombosis: characteristics of coronary atherosclerotic plaques underlying fatal occlusive thrombi. Br Heart $\mathcal{F} 1983 ; 50: 127-34$.

2 Davies M, Thomas A. Thrombosis and acute coronary artery lesions in sudden cardiac ischemic death. $N$ Engl $₹$ artery lesions in sudden

3 Davies MJ. A macro and micro view of coronary vascular insult in ischemic heart disease. Circulation 1990;82(suppl II):II-38-46

4 Qiao JH, Fishbein MC. The severity of coronary atherosclerosis at site of plaque rupture with occlusive thrombosis. $\mathscr{f}$ Am Coll Cardiol 1991;17:1138-42.

5 Fuster V, Badimon L, Badimon JJ, et al. The pathogenesis of coronary artery disease and the acute coronary syndromes. N Engl f Med 1992;326:242-50, 310-18.

6 Falk E, Shah PK, Fuster V. Coronary plaque disruption. Circulation 1995;92:656-71.

7 Lee RT, Libby P. The unstable atheroma. Arterioscler Thromb Vasc Biol 1997;17:1859-67.

8 Little WC, Constantinescu M, Applegate RJ, et al. Can coronary angiography predict the site of a subsequent myocardial infarction in patients with mild-to-moderate coronary dial infarction in patients with mild-to-moder

9 Mann JM, Davies. Vulnerable plaque: relation of characterMann JM, Davies. Vulnerable plaque: relation of character-
istics to degree of stenosis in human coronary arteries. Ciristics to degree of stenosis

10 Fishbein MC, Siegel RJ. How big are coronary atherosclerotic plaques that rupture? Circulation 1996;94:2662-6.

11 Mann JM, Davies MJ. Assessment of the severity of coronary artery disease at postmortem examination. Are the measurements clinically valid? Br Heart J 1995;74:528-30.

12 Glagov S, Weisenberg E, Zarins CK, et al. Compensatory enlargement of human atherosclerotic coronary arteries. $N$ Engl f Med 1987;316:1371-5.

13 McPherson DD, Sirna SJ, Hiratzka LF, et al. Coronary artery remodeling studied by high-frequency epicardial echocardiography: an early compensatory mechanism in patients with obstructive coronary atherosclerosis. 7 Am Coll Cardiol 1991;17:79-86.

$14 \mathrm{Ge} \mathrm{J}$, Erbel R, Zamorano J, et al. Coronary arterial remodeling in atherosclerotic disease: an intravascular

15 von Birgelen C, Airiian SG, Mintz GS, et al. Variations of remodeling in response to left main atherosclerosis assessed
with intravascular ultrasound in vivo. Am 7 Cardiol with intravascula

16 Sabate M, Kay P, de Feyter PJ, et al. Remodeling of atherosclerotic coronary arteries varies in relation to location and composition of plaque. Am f Cardiol 1999;84:135-40.

17 Varnava A. Coronary artery remodelling. Heart 1998;79: 109-10.

18 Di Mario C, Görge G, Peters R, et al, on behalf of the European Society of Cardiology. Clinical application and image interpretation in intracoronary ultrasound. Eur Heart $f$ 1998;19:207-29.

19 Erbel R, Ge J, Görge G, et al. Intravascular ultrasound classification of atherosclerotic lesions according to Ameri- can Heart Association recommendation. Coron Artery Dis 1999;10:489-99.

20 Pasterkamp G, Wensing PJW, Post MJ, et al. Paradoxical arterial wall shrinkage may contribute to luminal narrowing of human atherosclerotic femoral arteries. Circulation 1995;91:1444-9.

21 Nishioka T, Luo H, Eigler NL, et al. Contribution of inadequate compensatory enlargement to development of human coronary artery stenosis: an in vivo intravascular ultrasound study. $\mathcal{F}$ Am Coll Cardiol 1996;27:1571-6.

22 Mintz GS, Kent KM, Pichard AD, et al. Contribution of inadequate arterial remodeling to the development of focal coronary artery stenoses: an intravascular ultrasound study. Circulation 1997;95:1791-8.

23 Gussenhoven EJ, Geselschap JH, van Lankeren W, et al. Remodeling of atherosclerotic coronary arteries assessed with intravascular ultrasound in vitro. $\mathrm{Am} f \mathrm{Cardiol}$ 1997;79:699-702.

24 von Birgelen C, Mintz GS, de Vrey EA, et al. Atherosclerotic coronary lesions with inadequate compensatory enlargement have smaller plaque and vessel volumes: observations with three-dimensional intravascular ultrasound in vivo. Heart 1998;79:137-43.

25 Smits PC, Bos L, van Ufford MAQ, et al. Shrinkage of human coronary arteries is an important determinant of de human coronary arteries is an important determinant of de
novo atherosclerotic luminal stenosis: an in vivo intravascunovo atherosclerotic luminal stenosis:
lar ultrasound. Heart 1998;79:143-7.

26 Smits PC, Pasterkamp G, de Jaegere PPT, et al. Angioscopic complex lesions are predominantly compensatory enlarged: an angioscopic and intracoronary ultrasound study. Cardiovasc Res 1999;41:458-64.

27 Gyöngyösi M, Yang P, Hassan A, et al. Arterial remodelling of native human coronary arteries in patients with unstable angina pectoris: a prospective intravascular ultrasound angina pectoris: a prospective
study. Heart $1999 ; 82: 68-74$.

28 Zamorano J, Erbel R, Ge J, et al. Spontaneous plaque rupture visualized by intravascular ultrasound. Eur Heart 7 1994;15:131-3.

29 Bocksch WG, Schartl M, Beckmann SH, et al. Intravascular ultrasound imaging in patients with acute myocardial infarction: comparison with chronic stable angina pectoris. Coron Artery Dis 1994;5:727-35.

30 Baumgart D, Liu F, Haude M, et al. Acute plaque rupture and myocardial stunning in patient with normal coronary arteriography. Lancet 1995;346:193-4.

31 Ge J, Haude M, Görge G, et al. Silent healing of spontaneous plaque disruption demonstrated by intracoronary ultrasound. Eur Heart $\mathcal{F}$ 1995;16:1149-51.

32 Kearney P, Erbel R, Rupprecht HJ, et al. Differences in the morphology of unstable and stable coronary lesions and their impact on the mechanisms of angioplasty: an in vivo study with intravascular ultrasound. Eur Heart f 1996;17: study with

33 Moriuchi M, Saito S, Takaiwa Y, et al. Assessment of plaque rupture by intravascular ultrasound. Heart Vessels 1997; 12(suppl): 178-81.

$34 \mathrm{Ge}$ J, Chirillo F, Schwedtmann J, et al. Screening of ruptured plaques in patients with coronary artery disease by intravascular ultrasound. Heart 1999;81:621-7.

35 Falk E. Why do plaques rupture? Circulation 1992;86(suppl III):III-30-42.

36 Baroldi G, Silver MD, Mariani F, et al Correlation of morphological variables in the coronary atherosclerotic plaque with clinical patterns of ischemic heart disease. Am f Cardiovasc Pathol 1988;2:159-72.

37 Hangartner JRW, Charleston AJ, Davies MJ, et al. Morphological characteristics of clinically significant coronary artery stenosis in stable angina. Br Heart f 1986;56:501-8.

38 Pasterkamp G, Schoneveld AH, van der Wal AC, et al. Relation of arterial geometry to luminal narrowing and histologic markers for plaque vulnerability: the remodeling paradox. F Am Coll Cardiol 1998:32:655-62. 